\title{
On Decidability of Intermediate Levels of Concatenation Hierarchies
}

\author{
Jorge Almeida ${ }^{1}$, Jana Bartoňová ${ }^{2}$, Ondřej Klíma², and Michal Kunc ${ }^{2}$ * \\ 1 CMUP, Dep. Matemática, Faculdade de Ciências, Universidade do Porto, \\ Rua do Campo Alegre 687, 4169-007 Porto, Portugal, jalmeida@fc.up.pt \\ 2 Department of Mathematics and Statistics, Masaryk University \\ Kotlářská 2, 61137 Brno, Czech Republic, \\ xbartonovaj, klima, kunc@math.muni.cz
}

\begin{abstract}
It is proved that in every concatenation hierarchy of regular languages, decidability of one of its half levels, obtained by polynomial closure, implies decidability of the intersection of the following half level with its complement. In terms of the quantifier-alternation hierarchy of sentences in the first-order logic of finite words, this means that decidability of (definability in) the $\Sigma_{n}$ fragment implies that of $\Delta_{n+1}$. In particular, the decidability of $\Delta_{5}$ is obtained.
\end{abstract}

\section{Introduction}

A remarkable connection between finite automata/regular language theory and logic was found by McNaughton and Papert [6], who proved that star-free languages are exactly those languages that are definable in first-order logic $\mathrm{FO}[<]$ of finite words. The decidability of star-freeness of a regular language is in turn due to Schützenberger [15], who provided a key connection with algebra by showing that star-free languages are those whose syntactic monoids are aperiodic.

Within the class of all star-free languages, Brzozowski and Cohen [5] defined in 1971 the so-called dot-depth hierarchy, based on the polynomial closure and Boolean closure operators. A long standing open question about this hierarchy is to algorithmically determine the minimum level in the hierarchy to which a given star-free language belongs. The logical significance of this problem was discovered by Thomas [18, who showed that a language belongs to the $n$th level of the natural variant of the dot-depth hierarchy known as the Straubing-Thérien hierarchy if and only if it is definable by a sentence with $n$ alternations of quantifiers. Moreover, the $\Sigma_{n}$ and $\Delta_{n}$ fragments of $\mathrm{FO}[<]$ form intermediate levels of the hierarchy, and correspond to the polynomial closure and unambiguous polynomial closure of the $(n-1)$ th level, respectively. The class of $\Sigma_{n}$-definable languages is often referred to as the $(n+1 / 2)$ th level of the hierarchy, while the

\footnotetext{
* The first author was partially supported by CMUP (UID/MAT/00144/2013), which is funded by FCT (Portugal) with national (MEC) and European structural funds through the programs FEDER, under the partnership agreement PT2020. The last two authors were supported by grant 15-02862S of the Czech Science Foundation.
} 
$\Delta_{n}$ fragment defines the intersection of this half-level with its complement. It is also worth mentioning that the connection between the original dot-depth hierarchy and the Straubing-Thérien hierarchy is well understood through the work of Straubing [16, which provides an algebraic transformation from the latter to the former which preserves decidability in both directions.

Depending on the choice of the lowest level in a concatenation hierarchy, one is led to consider different hierarchies. Pin, Straubing and Thérien [8] and Pin and Weil [10] described the algebraic counterpart of the unambiguous polynomial closure and polynomial closure operators using the Mal'cev product of pseudovarieties. These results (together with [14, Theorem 4.6.50]) show that decidability of any integer level in an arbitrary concatenation hierarchy implies decidability of the intersection of the next half-level with its complement.

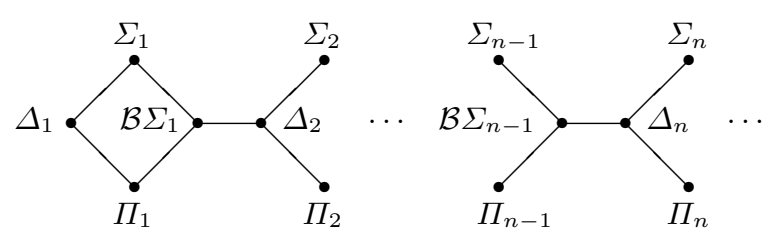

Fig. 1. The quantifier-alternation hierarchy of sentences of $\mathrm{FO}[<]$

The decidability of the dot-depth hierarchy turned out to be very difficult. In particular, the first algorithm for deciding membership of languages in the second level of the hierarchy has been announced only as late as 2014 by Place and Zeitoun 12. In the same paper, it is also shown that definability in $\Sigma_{3}$ is decidable (to which, for shortness, we will refer by saying $\Sigma_{3}$ is decidable) and decidability of $\Sigma_{4}$ was later also proved by Place [11. Place and Zeitoun [12] further provided, for each $n$, a non-effective description of languages definable in $\Delta_{n}$ based on inequalities valid in syntactic ordered monoids of languages definable in $\Sigma_{n-1}$. Their proof is specific for the Straubing-Thérien hierarchy, as it is based on manipulations with first-order formulas.

Thus, only a finite number of levels of the dot-depth hierarchy have been proved to be decidable. The main result of this paper, whose proof employs techniques of profinite monoids, implies the following statement:

For each $n$, the problem of definability in $\Delta_{n+1}$ polynomially reduces to the problem of definability in $\Sigma_{n}$. In particular, if $\Sigma_{n}$ is decidable, then so is $\Delta_{n+1}$.

More generally, we provide, for an arbitrary concatenation hierarchy, a polynomial time reduction of the membership problem for the intersection of any half-level with its complement to the membership problem for the previous halflevel. Combining with the results of Place and Zeitoun [12, one obtains that our result implies decidability of $\Delta_{4}$, a fact that was independently discovered by 
Place [11. Furthermore, from the decidability of $\Sigma_{4}$ also proved by Place [11, our result yields the decidability of $\Delta_{5}$.

Selected results on concatenation hierarchies are recalled in the following section, in order to fix terminology and notation; for a more comprehensive overview of results on these hierarchies and for a general introduction to the algebraic theory of regular languages, we refer to a handbook chapter by Pin 7 . Section 3 describes the relationship between intermediate levels in concatenation hierarchies, while Section 5 is devoted to turning this relationship into a polynomial time reduction of the membership problems.

\section{Overview of Results on Concatenation Hierarchies}

\subsection{Background and notation}

A binary relation $R$ on a set $M$ is said to be a quasiorder if it is reflexive and transitive. Every quasiorder $R$ on $M$ determines an equivalence relation $R^{\mathrm{e}}$ on $M$, consisting of all pairs $(s, t) \in R$ such that $(t, s) \in R$. Then $R$ induces a partial order on the quotient set $M / R^{\mathrm{e}}$, and we denote the resulting partially ordered set by $M / R$. The transitive closure of a binary relation $R$ on $M$ is denoted $\mathrm{T}(R)$.

A binary relation $R$ on a monoid $M$ is called stable if for all $(s, t) \in R$ and $z \in M$, both pairs $(s z, t z)$ and $(z s, z t)$ belong to $R$. If $R$ is a stable and reflexive relation on $M$, then its transitive closure $\mathrm{T}(R)$ is a stable quasiorder on $M$.

An ordered monoid $(M, \leqslant)$ is a monoid $M$ equipped with a stable partial order $\leqslant$. A homomorphism between ordered monoids $(M, \leqslant)$ and $(N, \leqslant)$ is a mapping $\varphi: M \rightarrow N$ which is a monoid homomorphism and at the same time monotone, i.e., for all $s, t \in M$ satisfying $s \leqslant t$, the inequality $\varphi(s) \leqslant \varphi(t)$ holds in $N$. Given a stable quasiorder $R$ on an unordered monoid $M$, the ordered set $M / R$ can be turned into an ordered monoid, and the natural projection $\pi: M \rightarrow M / R$ becomes a homomorphism of ordered monoids, with $M$ ordered by the equality relation.

A class of regular languages is a mapping $\mathcal{V}$ that associates with each finite alphabet $A$ a set of regular languages over $A$. A positive variety $\mathcal{V}$ is a class of regular languages such that each set $\mathcal{V}(A)$ contains $\emptyset$ and $A^{*}$ and is closed under finite intersections, finite unions and quotients, and additionally, for every homomorphism $f: A^{*} \rightarrow B^{*}$ and every $L \in \mathcal{V}(B)$, the language $f^{-1}(L)$ belongs to $\mathcal{V}(A)$. A positive variety $\mathcal{V}$ is a variety if each set $\mathcal{V}(A)$ is also closed under complementation. For a positive variety $\mathcal{V}$, we denote by Co- $\mathcal{V}$ the positive variety where each $\operatorname{Co-} \mathcal{V}(A)$ consists of all complements of languages in $\mathcal{V}(A)$.

\subsection{Concatenation Hierarchies}

For a set of languages $T$ over an alphabet $A$, its polynomial closure is the set of all languages over $A$, which are finite unions of languages of the form $L_{0} a_{1} L_{1} \ldots a_{n} L_{n}$, where $n \geqslant 0, a_{i} \in A$, and $L_{i} \in T$. If $\mathcal{V}$ is a variety of languages, then we denote by $\operatorname{Pol} \mathcal{V}$ the class of languages such that, for every alphabet $A$, 
the set $\operatorname{Pol} \mathcal{V}(A)$ is the polynomial closure of $\mathcal{V}(A)$. The resulting class $\operatorname{Pol} \mathcal{V}$ is a positive variety of languages (see Theorem 7.1 of [7]). Moreover, we denote by $\mathrm{BPol} \mathcal{V}$ the class of languages such that, for every alphabet $A$, the set $\operatorname{BPol} \mathcal{V}(A)$ is the closure of $\operatorname{Pol} \mathcal{V}(A)$ under all Boolean operations. In other words, $\mathrm{BPol} \mathcal{V}$ is the join of $\operatorname{Pol} \mathcal{V}$ and $\mathrm{Co}-\mathrm{Pol} \mathcal{V}$ in the lattice of all positive varieties.

A widely studied variant of the polynomial closure is the unambiguous polynomial closure UPol, which is known to satisfy $\mathrm{UPol} \mathcal{V}=\operatorname{Pol} \mathcal{V} \cap \mathrm{Co}-\mathrm{Pol} \mathcal{V}$ for every variety $\mathcal{V}$; for more details, see paragraph 7.2 of $[7$.

For a variety of languages $\mathcal{V}_{0}$, the concatenation hierarchy of basis $\mathcal{V}_{0}$ is a hierarchy of classes of languages defined by the rules $\mathcal{V}_{n+1 / 2}=\operatorname{Pol} \mathcal{V}_{n}$ and $\mathcal{V}_{n+1}=\mathrm{BPol} \mathcal{V}_{n}$, for every integer $n \geqslant 0$. Each half level in the hierarchy is a positive variety and each integer level is a variety. Such a hierarchy, together with complements of half levels, is depicted in Figure 2, which, except for $\mathcal{V}_{0}$,

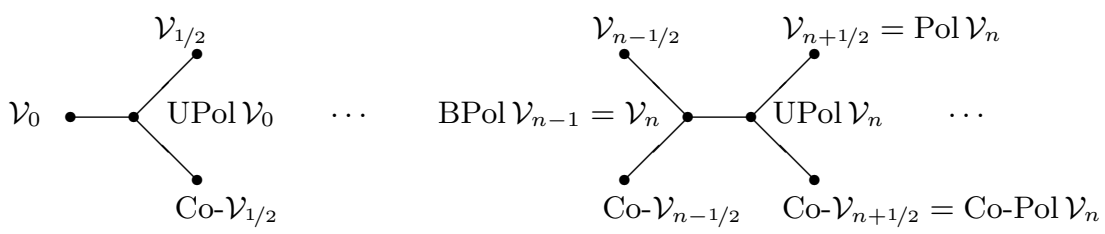

Fig. 2. A hierarchy of languages

is the language counterpart of Figure 1 in the case of the Straubing-Thérien hierarchy, whose basis is formed by the smallest variety of languages $\mathcal{V}_{0}$, with $\mathcal{V}_{0}(A)=\left\{\emptyset, A^{*}\right\}$ for each finite alphabet $A$. In the case of the Straubing-Thérien hierarchy, $\mathcal{V}_{0}=\mathrm{UPol} \mathcal{V}_{0}$ holds, and all other inclusions in the above picture are proper.

\subsection{Pseudovarieties and Pseudoidentities}

There is a one-to-one correspondence between varieties of languages and pseudovarieties of finite monoids, which are classes of finite monoids closed under homomorphic images, submonoids and finite direct products. Similarly, positive varieties of languages are in one-to-one correspondence with pseudovarieties of finite ordered monoids. For instance, a positive variety $\mathcal{V}$ corresponds to the pseudovariety $\mathrm{V}$ generated by syntactic ordered monoids of languages in $\mathcal{V}$. Then a language $L \subseteq A^{*}$ belongs to $\mathcal{V}$ if and only if its syntactic ordered monoid belongs to $\mathrm{V}$, while an ordered monoid $M$ belongs to $\mathrm{V}$ if and only if all preimages of upper sets in $M$ under homomorphisms $\varphi: A^{*} \rightarrow M$ belong to $\mathcal{V}(A)$. In particular, the decidability of the membership problems for $\mathcal{V}$ and for $\mathcal{V}$ are equivalent.

For an arbitrary pseudovariety $\mathrm{V}$ of ordered monoids, with the corresponding positive variety $\mathcal{V}$, the pseudovariety corresponding to the positive variety Co- $\mathcal{V}$ is $\bigvee^{\mathrm{d}}=\{(M, \geqslant) \mid(M, \leqslant) \in \mathrm{V}\}$. We denote by $\mathcal{B} \bigvee$ the join of $\mathrm{V}$ and its dual 
$\mathrm{V}^{\mathrm{d}}$ in the lattice of all pseudovarieties of ordered monoids; it is the pseudovariety corresponding to the Boolean closure of $\mathcal{V}$. The pseudovariety $\mathcal{B} \vee$ can be equivalently characterized as the pseudovariety of ordered monoids generated by the class $\{(M,=) \mid(M, \leqslant) \in \mathrm{V}\}$. We call a pseudovariety $\mathrm{V}$ of ordered monoids selfdual if $\mathrm{V}=\mathrm{V}^{\mathrm{d}}$; these are precisely pseudovarieties of the form $\mathcal{B W}$ for some pseudovariety $\mathrm{W}$, or equivalently, those which satisfy $\mathrm{V}=\mathcal{B} \mathrm{V}$. There is a oneto-one correspondence between selfdual pseudovarieties of ordered monoids and pseudovarieties of monoids; we will not distinguish between a selfdual pseudovariety $\mathrm{V}$ and the corresponding pseudovariety of monoids $\{M \mid(M, \leqslant) \in \mathrm{V}\}$.

Reiterman [13] (see [7, Section 4.1]) proved that every pseudovariety of monoids $\mathrm{V}$ can be characterized by some set of so-called pseudoidentities; each such set is usually called a basis of pseudoidentities for V. Pseudoidentities are generalizations of identities, that is, pairs $u=v$ of words $u, v \in A^{*}$, which determine equational axioms for classes of finite monoids. Since not all pseudovarieties of monoids are equational in this sense, Reiterman's idea was to consider identities of generalized words. For this purpose, one takes the completion $\widehat{A^{*}}$ of the free monoid $A^{*}$ with respect to the metric induced by homomorphisms from $A^{*}$ into finite monoids, in which, roughly speaking, two distinct words are very close if it takes a homomorphism from $A^{*}$ into a very large finite monoid to distinguish them. The metric monoid $\widehat{A^{*}}$ is profinite, which means that it is compact and each pair of its elements can be distinguished by a continuous homomorphism to a finite monoid endowed with the discrete topology. Moreover, the monoid $\widehat{A^{*}}$ is characterized by a universal property among profinite monoids, namely, for every mapping $\alpha: A \rightarrow S$ to a profinite monoid $S$, there exists a unique continuous homomorphism $\bar{\alpha}: \widehat{A^{*}} \rightarrow S$ extending $\alpha$ (see [2, Proposition 3.4]). This property can be in particular used if $S$ is a finite monoid.

A pseudoidentity $u=v$ is a pair of elements $u, v$ of $\widehat{A^{*}}$, and this pseudoidentity is satisfied by a finite monoid $M$ if $\bar{\alpha}(u)=\bar{\alpha}(v)$ holds for every mapping $\alpha: A \rightarrow M$. In the case of pseudovarieties of ordered monoids, the previous definition must be modified to use the so-called inequalities. An inequality $u \leqslant v$ is a pair of elements of $\widehat{A^{*}}$, and it is satisfied by an ordered monoid $(M, \leqslant)$ if $\bar{\alpha}(u) \leqslant \bar{\alpha}(v)$ holds for every mapping $\alpha: A \rightarrow M$. Note that in this paper, as usual in recent literature, the ordering of monoids corresponding to a positive variety of languages is dual to the one used by Pin 7], and thus all inequalities in [7] characterizing pseudovarieties of ordered monoids have to be reversed. This stems from the fact that the reverse of the syntactic order which used to appear in the literature has come to be preferred.

An element $e$ of a monoid $M$ is called an idempotent if $e e=e$; the set of all idempotents in $M$ is denoted by $E(M)$. For an arbitrary element $s$ of a profinite monoid $S$, the sequence $s^{n !}$ converges in $S$ to an idempotent, which is denoted by $s^{\omega}$. In particular, if $S$ is a finite monoid, then $s^{\omega}$ is the unique idempotent which is a power of $s$. We also write $s^{\omega-1}$ for the limit of the sequence $s^{n !-1}$. For every continuous homomorphism $\gamma: S \rightarrow T$ of profinite monoids and every $s \in S$, it is clear from the definition that $\gamma\left(s^{\omega}\right)=\gamma(s)^{\omega}$. A finite monoid is aperiodic if it 
satisfies the pseudoidentity $x^{\omega+1}=x^{\omega}$. The pseudovariety of aperiodic monoids corresponds to the variety of star-free languages (see [7, Theorem 5.2]).

\section{Bases of Inequalities for Polynomial Closure}

The aim of this section is to obtain suitable descriptions by inequalities of the various levels of concatenation hierarchies. Descriptions by arbitrary inequalities do not insure decidability as all pseudovarieties admit them.

We will rely on a result that gives the inequalities of the pseudovarieties corresponding to $\operatorname{Pol} \mathcal{V}$ and $\mathrm{UPol} \mathcal{V}$ as a function of those of the pseudovariety corresponding to $\mathcal{V}$ :

Proposition 1 ([8 9 10], [7, Theorems 6.5, 7.1 and 7.3]). Let $\mathrm{V}$ be a pseudovariety of monoids and $\mathcal{V}$ be the corresponding variety of languages. Then

(i) the pseudovariety of ordered monoids corresponding to the positive variety of languages Pol $\mathcal{V}$ is defined by the set of all inequalities $v^{\omega} \leqslant v^{\omega} u v^{\omega}$, where $u, v$ belong to $\widehat{A^{*}}$ for some finite set $A$ and $\vee$ satisfies $u=v$ and $v=v^{2}$,

(ii) the pseudovariety of monoids corresponding to the variety $\mathrm{UPol} \mathcal{V}$ is defined by the set of all pseudoidentities $v^{\omega}=v^{\omega} u v^{\omega}$, where $u, v$ belong to $\widehat{A}^{*}$ for some finite set $A$ and $\vee$ satisfies $u=v$ and $v=v^{2}$.

In the case of the Straubing-Thérien hierarchy, Place and Zeitoun [12] described a basis of inequalities for $\mathrm{V}_{n+1 / 2}$ very similar to the basis obtained from Proposition 11(i) by taking $\mathrm{V}$ equal to $\mathrm{V}_{n}$. The main difference is that instead of assuming that the pseudoidentity $u=v$ is valid in $\mathrm{V}_{n}$, it is required that the inequality $u \leqslant v$ holds in $\mathrm{V}_{n-1 / 2}$. A general relationship between such characterizations will now be explained using a new operator on pseudovarieties.

The inequalities of Proposition 1 may be interpreted in terms of Mal'cev products, an algebraic operation on pseudovarieties of monoids. Here we follow another route by defining a new operation on pseudovarieties uniquely in terms of inequalities.

For an arbitrary pseudovariety of ordered monoids $\mathrm{V}$, let $\mathrm{V}^{\mathrm{m}}$ denote the pseudovariety of ordered monoids with basis of inequalities consisting of all inequalities $v^{\omega} \leqslant v^{\omega} u v^{\omega}$, where $u, v \in \widehat{A^{*}}$ for some finite set $A$ and both the inequality $u \leqslant v$ and the pseudoidentity $v^{2}=v$ are valid in $\mathbf{V}$. The operator $\left({ }^{\prime}\right)^{\mathrm{m}}$ is monotone, that is, $\mathrm{V} \subseteq \mathrm{W}$ implies $\mathrm{V}^{\mathrm{m}} \subseteq \mathrm{W}^{\mathrm{m}}$, because every inequality from the basis for $\mathrm{W}^{\mathrm{m}}$ is included in the basis for $\mathrm{V}^{\mathrm{m}}$. Additionally, denote by $\mathrm{V}^{\mathrm{M}}$ the monoid pseudovariety $\mathrm{V}^{\mathrm{m}} \cap\left(\mathrm{V}^{\mathrm{m}}\right)^{\mathrm{d}}$. Then $\mathrm{V}^{\mathrm{M}}$ is defined by pseudoidentities of the form $v^{\omega}=v^{\omega} u v^{\omega}$, where $u, v \in \widehat{A^{*}}$ for some finite set $A$ and both the inequality $u \leqslant v$ and the pseudoidentity $v^{2}=v$ are valid in $\mathrm{V}$.

Remark. According to [7, Theorem 6.5], if $\vee$ is a selfdual pseudovariety, then both $\mathrm{V}^{\mathrm{m}}$ and $\mathrm{V}^{\mathrm{M}}$ can be expressed in terms of the Mal'cev product as $\mathrm{V}^{\mathrm{m}}=$ $\mathrm{W} m \mathrm{~V}$ and $\mathrm{V}^{\mathrm{M}}=\mathrm{LI}(\mathrm{m}) \mathrm{V}$, with $\mathrm{W}$ and $\mathrm{LI}$ pseudovarieties of finite semigroups defined by the inequality $x^{\omega} \leqslant x^{\omega} y x^{\omega}$ and the pseudoidentity $x^{\omega}=x^{\omega} y x^{\omega}$, respectively. However, if $\mathrm{V}$ is not selfdual, such results cannot be formulated, as the corresponding Mal'cev products are not defined. 
Lemma 2. Every pseudovariety of ordered monoids $\mathrm{V}$ satisfies the inclusion $\mathrm{V}^{\mathrm{d}} \subseteq \mathrm{V}^{\mathrm{m}}$. In particular, if $\mathrm{V}$ is selfdual, then $\mathrm{V} \subseteq \mathrm{V}^{\mathrm{m}}$.

Proof. It has to be verified that every ordered monoid $M \in \mathrm{V}^{\mathrm{d}}$ satisfies all inequalities $v^{\omega} \leqslant v^{\omega} u v^{\omega}$, with $u \leqslant v$ and $v^{2}=v$ valid in $\mathrm{V}$. However, the assumptions on $u$ and $v$ imply that $v \leqslant u$ and $v^{2}=v$ are satisfied by $M$. Then $M$ satisfies also $v^{\omega}=v^{\omega} v v^{\omega} \leqslant v^{\omega} u v^{\omega}$.

The following lemma provides a condition on a pseudovariety $\mathrm{V}$ which guarantees that the pseudovariety $(\mathcal{B V})^{\mathrm{m}}$ can be obtained by applying the operator $\left(\_\right)^{\mathrm{m}}$ directly to $\mathrm{V}$.

Lemma 3. Let $\mathrm{V}=\mathrm{W}^{\mathrm{m}}$, with $\mathrm{W}$ a selfdual pseudovariety of ordered monoids. Then $\mathrm{V}^{\mathrm{m}}=(\mathcal{B} \bigvee)^{\mathrm{m}}$ and $\mathrm{V}^{\mathrm{M}}=(\mathcal{B} \mathrm{\vee})^{\mathrm{M}}$.

Proof. Clearly, the first equality implies the second one. Since the operator $\left(\_\right)^{\mathrm{m}}$ is monotone, we have $\mathrm{V}^{\mathrm{m}} \subseteq(\mathcal{B V})^{\mathrm{m}}$. It remains to prove that every inequality from the basis for $\mathrm{V}^{\mathrm{m}}$ is valid in $(\mathcal{B} \mathrm{V})^{\mathrm{m}}$ as well. Let $u, v \in \widehat{A^{*}}$ be such that $u \leqslant v$ and $v^{2}=v$ are valid in $\mathrm{V}$. Since the inequality $v^{\omega} \leqslant v^{\omega} u v^{\omega}$ is valid in $\mathrm{V}^{\mathrm{m}}$, it holds also in $\mathrm{V}^{\mathrm{d}}$ by Lemma 2 , which means that $\mathrm{V}$ satisfies $v^{\omega} u v^{\omega} \leqslant v^{\omega}$. On the other hand, because $\mathrm{W}$ is selfdual by assumption, the inclusion $\mathrm{W} \subseteq \mathrm{V}$ holds by Lemma 2 and consequently both $u \leqslant v$ and $v^{2}=v$ are also valid in $\mathrm{W}$. Hence, the pseudovariety $\mathrm{V}=\mathrm{W}^{\mathrm{m}}$ satisfies $v^{\omega} \leqslant v^{\omega} u v^{\omega}$ too. Together, this shows that the pseudoidentity $v^{\omega} u v^{\omega}=v^{\omega}$ is valid in $\mathrm{V}$, and consequently it is also valid in $\mathcal{B} \vee$. Now consider elements $\bar{u}=v^{\omega} u v^{\omega}$ and $\bar{v}=v^{\omega}$ of $\widehat{A^{*}}$. Then both $\bar{u}=\bar{v}$ and $\bar{v}^{2}=\bar{v}$ hold in $\mathcal{B}$. This implies that the inequality $\bar{v}^{\omega} \leqslant \bar{v}^{\omega} \bar{u} \bar{v}^{\omega}$ is valid in $(\mathcal{B} \bigvee)^{\mathrm{m}}$. However, this inequality is equivalent to $v^{\omega} \leqslant v^{\omega} u v^{\omega}$, because $\left(v^{\omega}\right)^{\omega}=v^{\omega}$ and $v^{\omega} v^{\omega}=v^{\omega}$ hold in $\widehat{A^{*}}$. Hence, the inequality $v^{\omega} \leqslant v^{\omega} u v^{\omega}$ is valid in $(\mathcal{B V})^{\mathrm{m}}$.

Lemma 3 has the following consequence for concatenation hierarchies.

Proposition 4. Let $\left(\mathcal{V}_{k}\right)_{k \in \mathbb{N} / 2}$ be an arbitrary concatenation hierarchy and let $\left(\mathrm{V}_{k}\right)_{k \in \mathbb{N} / 2}$ be the corresponding hierarchy of pseudovarieties of ordered monoids. Then, for each positive integer $n$, the following equalities hold:

$$
\mathrm{V}_{n+1 / 2}=\left(\mathrm{V}_{n}\right)^{\mathrm{m}}=\left(\mathrm{V}_{n-1 / 2}\right)^{\mathrm{m}} \text {. }
$$

Proof. The equality $\mathrm{V}_{n+1 / 2}=\left(\mathrm{V}_{n}\right)^{\mathrm{m}}$ comes from Proposition 11(i). The equality $\left(\mathrm{V}_{n}\right)^{\mathrm{m}}=\left(\mathrm{V}_{n-1 / 2}\right)^{\mathrm{m}}$ follows from Lemma 3 , because of $\mathrm{V}_{n-1 / 2}=\left(\mathrm{V}_{n-1}\right)^{\mathrm{m}}$ and $\mathrm{V}_{n}=\mathcal{B} \bigvee_{n-1 / 2}$.

\section{Effectively testing inequalities}

It is not clear that the inequalities defining $\mathrm{V}^{\mathrm{m}}$ may be effectively tested in a given ordered monoid $(M, \leqslant)$. The aim of this section is to solve this problem. For this purpose, we start by defining a relation determined by inequalities that 
hold in $\mathrm{V}$. This relation allows to identify for which pairs $(s, t)$ of elements of $M$ the inequality $t \leqslant t s t$ should be tested in order to assure that $M$ belongs to $\mathrm{V}^{\mathrm{m}}$.

Recall that for a mapping $\alpha: A \rightarrow M$, there is a unique continuous homomorphism $\bar{\alpha}:\left(\widehat{A^{*}},=\right) \rightarrow(M, \leqslant)$ that extends $\alpha$. We define a relation $\sigma \vee(M)$ on $M$ as the set of all pairs $(\bar{\alpha}(u), \bar{\alpha}(v))$, where $\alpha: A \rightarrow M$ is a mapping from an arbitrary finite alphabet $A$, and $u, v \in \widehat{A^{*}}$ are such that the inequality $u \leqslant v$ holds in $\mathrm{V}$. Thus, $\sigma_{\mathrm{V}}(M)$ is an order analog of the 2-pointlike pair relation on a finite monoid, which in turn may be viewed as a topological separation problem on regular languages within the free profinite monoid $\widehat{A^{*}}[1$. In the work of Place and Zeitoun [12] an element of $\sigma_{\bigvee_{3 / 2}}(M)$ is called a two-element $\Sigma_{2}$-chain of $M$, and may also be viewed as a topological separation property.

The relation $\sigma_{\mathrm{V}}(M)$ is obviously reflexive. It is also stable, since for given $(\bar{\alpha}(u), \bar{\alpha}(v)) \in \sigma_{\mathrm{V}}(M)$ and $z \in M$, the pairs $(\bar{\alpha}(u) z, \bar{\alpha}(v) z)$ and $(z \bar{\alpha}(u), z \bar{\alpha}(v))$ can be shown to belong to $\sigma \vee(M)$ by choosing a new letter $x \notin A$, observing that $u x \leqslant v x$ and $x u \leqslant x v$ hold in $\mathrm{V}$, and extending $\alpha$ by setting $\alpha(x)=z$. However, it need not be transitive. The following lemma shows that instead of using all possible mappings $\alpha$, it is sufficient to use an arbitrary surjective mapping.

Lemma 5. Let $\mathrm{V}$ be a pseudovariety of ordered monoids and $(M, \leqslant)$ a finite ordered monoid. Let $\alpha: A \rightarrow M$ be an arbitrary surjective mapping. Then, for every $s, t \in M$, the pair $(s, t)$ belongs to $\sigma_{\mathrm{V}}(M)$ if and only if $(s, t)=(\bar{\alpha}(u), \bar{\alpha}(v))$ for some $u, v \in \widehat{A^{*}}$ such that the inequality $u \leqslant v$ holds in $\mathrm{V}$.

Proof. The "if" statement is trivial. In order to prove the "only if" part, assume that $(s, t) \in \sigma_{\mathrm{V}}(M)$, that is, there exists a mapping $\beta: B \rightarrow M$ such that $(s, t)=$ $(\bar{\beta}(u), \bar{\beta}(v))$ for some $u, v \in \widehat{B^{*}}$, with $u \leqslant v$ valid in $\mathrm{V}$. The universal property of $\widehat{B^{*}}$ and surjectivity of $\alpha$ imply that there exists a continuous homomorphism $\gamma: \widehat{B^{*}} \rightarrow \widehat{A^{*}}$ such that $\bar{\beta}=\bar{\alpha} \circ \gamma$. Then $\gamma(u) \leqslant \gamma(v)$ holds in $\mathrm{V}$, and $(s, t)=$ $(\bar{\alpha}(\gamma(u)), \bar{\alpha}(\gamma(v)))$.

Based on the relation $\sigma_{\vee}(M)$, an alternative characterization of ordered monoids $(M, \leqslant)$ belonging to the pseudovariety $\mathrm{V}^{\mathrm{m}}$ can be formulated.

Lemma 6. Let $\mathrm{V}$ be a pseudovariety of ordered monoids and $(M, \leqslant)$ a finite ordered monoid. Then $(M, \leqslant) \in \mathrm{V}^{\mathrm{m}}$ if and only if $t \leqslant$ tst holds for every pair of elements $s, t \in M$ such that $(s, t) \in \sigma_{\mathrm{V}}(M)$ and $t \in E(M)$.

Proof. Assume that $(M, \leqslant)$ belongs to $\mathrm{V}^{\mathrm{m}}$ and let $(s, t) \in \sigma_{\mathrm{V}}(M)$ be a pair of elements of $M$ such that $t^{2}=t$. Then there exist $u, v \in \widehat{A^{*}}$ such that the inequality $u \leqslant v$ holds in $\mathrm{V}$ and $\bar{\alpha}(u)=s$ and $\bar{\alpha}(v)=t$ for a certain mapping $\alpha: A \rightarrow M$. Multiplying the inequality $u \leqslant v$ by $v^{\omega-1}$, one obtains that the inequality $u v^{\omega-1} \leqslant v^{\omega}$ is also valid in $\mathrm{V}$. Denoting $\bar{u}=u v^{\omega-1} \in \widehat{A^{*}}$ and $\bar{v}=$ $v^{\omega} \in \widehat{A^{*}}$, both the inequality $\bar{u} \leqslant \bar{v}$ and the pseudoidentity $\bar{v}^{2}=\bar{v}$ are valid in $\mathrm{V}$. Therefore, the inequality $\bar{v}^{\omega} \leqslant \bar{v}^{\omega} \bar{u} \bar{v}^{\omega}$ belongs to the basis of inequalities for $\mathrm{V}^{\mathrm{m}}$. This implies that $\bar{\alpha}\left(\bar{v}^{\omega}\right) \leqslant \bar{\alpha}\left(\bar{v}^{\omega} \bar{u} \bar{v}^{\omega}\right)$, because $(M, \leqslant) \in \mathrm{V}^{\mathrm{m}}$. Now we simply compute that $\bar{\alpha}\left(\bar{v}^{\omega}\right)=\bar{\alpha}\left(v^{\omega}\right)=\bar{\alpha}(v)^{\omega}=t^{\omega}=t$ and $\bar{\alpha}(\bar{u})=\bar{\alpha}\left(u v^{\omega-1}\right)=$ 
$\bar{\alpha}(u) \bar{\alpha}(v)^{\omega-1}=s t^{\omega-1}=s t$. Then the inequality $\bar{\alpha}\left(\bar{v}^{\omega}\right) \leqslant \bar{\alpha}\left(\bar{v}^{\omega} \bar{u} \bar{v}^{\omega}\right)$ translates as $t \leqslant t s t t=t s t$, which proves the direct part of the statement.

To prove the converse, assume that $t \leqslant t s t$ holds for every pair of elements $s, t \in M$ such that $(s, t) \in \sigma_{\vee}(M), t \in E(M)$. Let $u, v \in \widehat{A^{*}}$, for a finite alphabet $A$, be such that $u \leqslant v$ and $v^{2}=v$ are valid in $\mathrm{V}$. It has to be shown that $M$ satisfies the inequality $v^{\omega} \leqslant v^{\omega} u v^{\omega}$. Let $\alpha: A \rightarrow M$ be an arbitrary mapping. Since $u \leqslant v$ and $v^{2}=v$ hold in $\mathrm{V}$, the inequality $v^{\omega} u v^{\omega} \leqslant v^{\omega}$ is also valid in $\mathrm{V}$. Consider elements $s=\bar{\alpha}\left(v^{\omega} u v^{\omega}\right)$ and $t=\bar{\alpha}\left(v^{\omega}\right)$ of $M$. Then $(s, t)$ belongs to $\sigma_{\vee}(M)$ and $t$ is an idempotent. Hence, by assumption, the inequality $t \leqslant t s t$ holds in $(M, \leqslant)$, which means that $\bar{\alpha}\left(v^{\omega}\right) \leqslant \bar{\alpha}\left(v^{\omega}\right) \bar{\alpha}\left(v^{\omega} u v^{\omega}\right) \bar{\alpha}\left(v^{\omega}\right)=\bar{\alpha}\left(v^{\omega} u v^{\omega}\right)$. This proves that $(M, \leqslant)$ satisfies the inequality $v^{\omega} \leqslant v^{\omega} u v^{\omega}$.

An analogous characterization of the pseudovariety $\mathrm{V}^{\mathrm{M}}$ follows directly from the characterizations of $\mathrm{V}^{\mathrm{m}}$ and $\left(\mathrm{V}^{\mathrm{m}}\right)^{\mathrm{d}}$ obtained by Lemma 6

Corollary 7. Let $\mathrm{V}$ be a pseudovariety of ordered monoids and $M$ a finite monoid. Then $M \in \mathrm{V}^{\mathrm{M}}$ if and only if $t=$ tst holds for every pair of elements $s, t \in M$ such that $(s, t) \in \sigma_{\mathrm{V}}(M)$ and $t \in E(M)$.

We return now to concatenation hierarchies with an application of Lemma 6 that is now obtained directly from Proposition 4 .

Proposition 8. Let $\left(\mathcal{V}_{k}\right)_{k \in \mathbb{N} / 2}$ be an arbitrary concatenation hierarchy of languages and let $\left(\mathrm{V}_{k}\right)_{k \in \mathbb{N} / 2}$ be the corresponding hierarchy of pseudovarieties of ordered monoids. Let $(M, \leqslant)$ be a finite ordered monoid. Then, for every positive integer $n$, the following conditions are equivalent:

(i) $(M, \leqslant) \in \mathrm{V}_{n+1 / 2}$,

(ii) $M$ satisfies $t \leqslant t$ st for all $(s, t) \in \sigma_{\mathrm{\vee}_{n}}(M), t \in E(M)$,

(iii) $M$ satisfies $t \leqslant$ tst for all $(s, t) \in \sigma_{\mathrm{V}_{n-1 / 2}}(M), t \in E(M)$.

In the case of star-free languages, Proposition 8 can be formulated in a slightly different way; this formulation in particular shows that Proposition 8 generalizes Theorem 7 of Place and Zeitoun 12 from the case of the Straubing-Thérien hierarchy to an arbitrary concatenation hierarchy.

Corollary 9. Let $\left(\mathcal{V}_{k}\right)_{k \in \mathbb{N} / 2}$ be an arbitrary concatenation hierarchy of star-free languages, with corresponding hierarchy of pseudovarieties of aperiodic ordered monoids $\left(\mathrm{V}_{k}\right)_{k \in \mathbb{N} / 2}$. Let $(M, \leqslant)$ be a finite ordered aperiodic monoid. Then, for every positive integer $n$, the following conditions are equivalent:

(i) $(M, \leqslant) \in \mathrm{V}_{n+1 / 2}$,

(ii) $M$ satisfies $t^{\omega} \leqslant t^{\omega}$ st $t^{\omega}$ for all $(s, t) \in \sigma_{\mathrm{V}_{n}}(M)$,

(iii) $M$ satisfies $t^{\omega} \leqslant t^{\omega} s t^{\omega}$ for all $(s, t) \in \sigma_{\mathrm{V}_{n-1 / 2}}(M)$.

Proof. First, it will be proved that (i) implies (ii). Assume that (i) is true. Then condition (ii) of Proposition 8 is satisfied. In order to prove (ii) assume that $(s, t) \in \sigma \mathrm{\vee}_{n}(M)$. Since $\sigma_{\mathrm{V}_{n}}(M)$ is a stable relation, we have $\left(t^{\omega} s t^{\omega}, t^{\omega+1}\right) \in$ 
$\sigma_{\vee_{n}}(M)$. As $t^{\omega+1}=t^{\omega}$ due to aperiodicity of $M$, condition (ii) of Proposition 8 can be applied to the pair $\left(t^{\omega} s t^{\omega}, t^{\omega}\right) \in \sigma_{\vee_{n}}(M)$, which shows that $t^{\omega} \leqslant t^{\omega} t^{\omega} s t^{\omega} t^{\omega}=t^{\omega} s t^{\omega}$ holds in $(M, \leqslant)$. This proves that (ii) is true. In order to verify the equivalence of (i) and (ii), it is now sufficient to observe that (ii) obviously implies condition (ii) of Proposition 8

In the same way, one can prove the equivalence of (iii) and condition (iii) of Proposition 8 , which finishes the proof.

Theorem 7 of [12] proves the equivalence of conditions (i) and (iii) for the Straubing-Thérien hierarchy. One of the contributions of $[\overline{12}$ is an algorithm for computing the relation $\sigma_{\mathrm{V}}(M)$ in the case of $\mathrm{V}$ corresponding to the level $3 / 2$ of this hierarchy, which directly implies decidability of $\mathrm{V}_{5 / 2}$ and $\mathrm{V}_{5 / 2} \cap\left(\mathrm{V}_{5 / 2}\right)^{\mathrm{d}}$. In this paper, rather than computing directly the relation $\sigma_{\mathrm{V}}(M)$, we attempt to prove decidability of pseudovarieties by computing the transitive closure of $\sigma_{\mathrm{V}}(M)$. The following section shows that this approach can be used to verify validity of all equalities $t=t s t$ in Corollary 7 . Although one needs to be able to verify all inequalities $t \leqslant t s t$ in order to decide membership in the pseudovariety $\mathrm{V}^{\mathrm{m}}$, dealing with equalities is sufficient to obtain decidability of the pseudovariety $\mathrm{V}^{\mathrm{M}}$.

\section{Polynomial Reduction of the Membership Problem}

Let $M$ be a finite monoid and let $\mathrm{V}$ be a pseudovariety of ordered monoids. We say that a stable quasiorder $\rho$ on $M$ is a $\mathrm{V}$-quasiorder if the quotient ordered monoid $M / \rho$ belongs to $\mathrm{V}$. For a pair of stable quasiorders $\rho$ and $\tau$, the relation $\rho \cap \tau$ is a stable quasiorder as well, and the quotient $M /(\rho \cap \tau)$ is isomorphic to a submonoid of the ordered monoid $M / \rho \times M / \tau$. This shows that the set of all $\mathrm{V}$-quasiorders of the monoid $M$ is closed under intersection, and consequently there exists the smallest $\mathrm{V}$-quasiorder on $M$, denoted $\rho_{\mathrm{V}}(M)$. If the pseudovariety $\mathrm{V}$ is decidable, then the relation $\rho_{\mathrm{V}}(M)$ is computable, since there are only finitely many binary relations on the finite set $M$.

Lemma 10. Let $M$ be a finite monoid and let $\mathrm{V}$ be a pseudovariety of ordered monoids. Then $\rho_{\mathrm{V}}(M)$ is equal to the transitive closure of $\sigma_{\mathrm{V}}(M)$.

Proof. For every mapping $\alpha: A \rightarrow M$, the composition of $\bar{\alpha}$ with the natural projection $\pi: M \rightarrow M / \rho_{\mathrm{V}}(M) \in \mathrm{V}$ satisfies $\pi \bar{\alpha}(u) \leqslant \pi \bar{\alpha}(v)$ for every inequality $u \leqslant v$ valid in $\mathrm{V}$. This shows that $\sigma_{\mathrm{V}}(M) \subseteq \rho_{\mathrm{V}}(M)$, and consequently $\mathrm{T}\left(\sigma_{\mathrm{V}}(M)\right) \subseteq \rho_{\mathrm{V}}(M)$ holds, as $\rho_{\mathrm{V}}(M)$ is transitive.

Conversely, because $\sigma_{\mathrm{V}}(M)$ is a stable reflexive relation on $M$, its transitive closure $\tau=\mathrm{T}\left(\sigma_{\mathrm{V}}(M)\right)$ is a stable quasiorder on $M$. Therefore, in order to prove that $\tau \supseteq \rho_{\mathrm{V}}(M)$, it suffices to verify that the ordered monoid $M / \tau$ belongs to $\mathrm{V}$. So, let $u \leqslant v$ be an arbitrary inequality that holds in $\mathrm{V}$, with $u, v \in \widehat{A^{*}}$, and consider any mapping $\beta: A \rightarrow M / \tau$. Let $\alpha: A \rightarrow M$ be any mapping such that $\beta=\pi \circ \alpha$, where $\pi: M \rightarrow M / \tau$ denotes the natural projection. Then the unique extensions of the mappings $\alpha$ and $\beta$ to the monoid $\widehat{A^{*}}$ satisfy $\bar{\beta}=\pi \circ \bar{\alpha}$. By 
definition of the relation $\sigma_{\mathrm{V}}(M)$, the pair $(\bar{\alpha}(u), \bar{\alpha}(v))$ belongs to $\sigma_{\mathrm{V}}(M)$, and consequently also to $\tau$. Altogether, we obtain $\bar{\beta}(u)=\pi(\bar{\alpha}(u)) \leqslant \pi(\bar{\alpha}(v))=\bar{\beta}(v)$, thus showing that $u \leqslant v$ is satisfied by $M / \tau$.

The following lemma, analogous to Lemma 4.1 of 4 , shows that when verifying the condition of Corollary 7 that is, the condition

$$
(\forall s, t \in M)\left(\left((s, t) \in R \wedge t^{2}=t\right) \Longrightarrow t s t=t\right),
$$

one can make use of the relation $\rho_{\mathrm{V}}(M)$ for $R$ instead of $R=\sigma_{\mathrm{V}}(M)$.

Lemma 11. Let $M$ be a finite monoid and let $R$ be a reflexive and stable binary relation on $M$. Then $R$ satisfies (1) if and only if the transitive closure of $R$ satisfies (1).

Proof. Assume that $t=t s t$ is satisfied for each pair $(s, t) \in R$ such that $t^{2}=t$. We show by induction with respect to $n$ that $t=t s t$ holds also for each pair $(s, t)$ from $R^{n}$, with $t \in E(M)$. For $n=1$, this is the assumption. Let $n>1$ and let $(s, t) \in R^{n}$ be an arbitrary pair such that $t^{2}=t$. Thus, there exists $z \in M$ such that $(s, z) \in R$ and $(z, t) \in R^{n-1}$. Since $R$ is stable, we have $(t s t, t z t) \in R$. The induction assumption gives $t=t z t$, which means that $(t s t, t) \in R$. Then $t=t(t s t) t=t s t$ by the assumption on $R$, as required. As the transitive closure of $R$ is the union of all relations $R^{n}$, the statement is proved.

In order to construct a polynomial time reduction of the membership problem for the pseudovariety $\mathrm{V}^{\mathrm{M}}$ to that of $\mathrm{V}$, we proceed in a way similar to 14, Subsection 4.6.2], where the membership problem for some Mal'cev products is solved by constructing certain congruences on a monoid $M$, and testing whether the quotient monoid belongs to $\mathrm{V}$. However, in our case, as $\mathrm{V}$ is a variety of ordered monoids, instead of a congruence, an appropriate stable quasiorder has to be constructed.

Let $M$ be a finite monoid. Denote by $\kappa(M)$ the union of all stable relations on $M$ satisfying (1). Then $\kappa(M)$ is obviously also a stable relation satisfying (1). Moreover, it is reflexive, as the identity relation has these properties, and it is transitive, as the transitive closure of a stable relation satisfying (1) is such a relation as well, by Lemma 11. This shows that $\kappa(M)$ is a quasiorder on $M$.

For a given pseudovariety of ordered monoids $\mathrm{V}$, let $\mathrm{V}^{\kappa}$ be the class of all monoids $M$ such that $M / \kappa(M) \in \mathrm{V}$.

Lemma 12. Let $\mathrm{V}$ be a pseudovariety of ordered monoids. Then $\mathrm{V}^{\kappa}=\mathrm{V}^{\mathrm{M}}$. In particular, $\mathrm{V}^{\kappa}$ is a pseudovariety of monoids.

Proof. Corollary 7 shows that $M$ belongs to $\mathrm{V}^{\mathrm{M}}$ if and only if $\sigma \mathrm{\vee}(M)$ satisfies (1), which holds precisely when $\rho_{\mathrm{V}}(M)$ satisfies (1), according to Lemmata 10 and 11 . This is equivalent to the requirement $\rho_{\mathrm{V}}(M) \subseteq \kappa(M)$, which holds if and only if $M / \kappa(M) \in \mathrm{V}$, by definition of $\rho_{\mathrm{V}}(M)$. The latter condition is exactly the definition of membership of $M$ in $\mathrm{V}^{\kappa}$. 
The algorithm for deciding membership in $\mathrm{V}^{\mathrm{M}}$ is based on calculating the relation $\kappa(M)$.

Lemma 13. For an arbitrary finite monoid $M$, the quasiorder $\kappa(M)$ can be calculated in polynomial time.

Proof. For every idempotent $e \in E(M)$, let $\kappa_{e}$ be the binary relation on $M$ defined by the rule

$$
(a, b) \in \kappa_{e} \Longleftrightarrow(\forall p, q \in M)(p b q=e \Longrightarrow e \cdot p a q \cdot e=e) .
$$

Each of the relations $\kappa_{e}$ can be calculated in polynomial time. It will be proved that the relation $\kappa(M)$ is equal to the intersection of all relations $\kappa_{e}$, which means that it can be calculated in polynomial time as well.

In order to show that $\kappa(M) \subseteq \kappa_{e}$ for every $e \in E(M)$, let $(a, b) \in \kappa(M)$ and $p, q \in M$ be such that $p b q=e$. The stability of $\kappa(M)$ implies that $(p a q, p b q) \in$ $\kappa(M)$, and since $p b q=e \in E(M)$, condition (1) gives $e \cdot p a q \cdot e=e$.

Conversely, in order to prove that the relation $\bigcap_{e \in E(M)} \kappa_{e}$ is contained in $\kappa(M)$, it is sufficient to verify that $\bigcap_{e \in E(M)} \kappa_{e}$ is a stable relation satisfying (1). The stability of the intersection follows directly from the stability of each $\kappa_{e}$, which is obvious from the definition. In order to verify condition (1), let $(s, t)$ belong to all relations $\kappa_{e}$, with $t \in E(M)$. In particular, it belongs to $\kappa_{t}$, which gives $t s t=t$ by choosing $p=q=1$.

The desired polynomial reduction of the membership problem for $\mathrm{V}^{\mathrm{M}}$ is obtained directly by combining Lemmata 12 and 13 and the definition of $\mathrm{V}^{\kappa}$.

Proposition 14. For every pseudovariety of ordered monoids $\mathrm{V}$, the membership problem for the pseudovariety $\mathrm{V}^{\mathrm{M}}$ can be reduced in polynomial time to the membership problem for $\mathrm{V}$.

Note that for an arbitrary variety of languages $\mathcal{V}$, with the corresponding pseudovariety of monoids V, Proposition 1|(ii) states that the pseudovariety corresponding to the variety $\mathrm{UPol} \mathcal{V}$ is precisely $\mathrm{V}^{\mathrm{M}}$. Therefore, as a special case of Proposition 14 we obtain the known result that decidability of the variety $\mathcal{V}$ implies decidability of the variety $\operatorname{UPol} \mathcal{V}$ (see [14, Theorem 4.6.50]).

Propositions 4 and 14 together give the main result of this paper:

Theorem 15. Let $\left(\mathcal{V}_{k}\right)_{k \in \mathbb{N} / 2}$ be an arbitrary concatenation hierarchy of regular languages and let $\left(\mathrm{V}_{k}\right)_{k \in \mathbb{N} / 2}$ be the corresponding hierarchy of pseudovarieties of ordered monoids. If $\mathrm{V}_{n-1 / 2}$ is a decidable pseudovariety, then $\mathrm{V}_{n+1 / 2} \cap\left(\mathrm{V}_{n+1 / 2}\right)^{\mathrm{d}}$ is also decidable, that is, the variety $\mathrm{UPol}_{n}$ is decidable.

Using decidability of the variety $\mathcal{V}_{7 / 2}$ proved by Place [11, this theorem gives

Corollary 16. It is algorithmically decidable whether a given regular language is definable in $\Delta_{5}$. 


\section{References}

1. J. Almeida: Some algorithmic problems for pseudovarieties, Publ. Math. Debrecen 54 Suppl. (1999), 531-552.

2. J. Almeida: Profinite semigroups and applications. In Structural Theory of Automata, Semigroups and Universal Algebra. V.B. Kudryavtsev and I.G. Rosenberg eds., Springer, 2005, 1-45.

3. J. Almeida, O. Klíma: A counterexample to a conjecture concerning concatenation hierarchies. Inform. Process. Lett. 110 (2009), 4-7.

4. J. Almeida, O. Klíma: New decidable upper bound of the second level in the Straubing-Thérien concatenation hierarchy of star-free languages. Discrete Math. Theor. Comput. Sci. 12 (2010), 41-58.

5. J.A. Brzozowski, R.S. Cohen: Dot-depth of star-free events. J. Comput. System Sci. 5 (1971), 1-15.

6. R. McNaughton, S. Papert: Counter-Free Automata. MIT Press, 1971.

7. J.-É. Pin: Syntactic semigroups, Chapter 10 in Handbook of Formal Languages. G. Rozenberg and A. Salomaa eds., Springer, 1997.

8. J.-É. Pin, H. Straubing, D. Thérien: Locally trivial categories and unambiguous concatenation. J. Pure Appl. Algebra 52 (1988), 297-311.

9. J.-É. Pin, P. Weil: Profinite semigroups, Mal'cev products and identities. J. Algebra 182 (1996), 604-626.

10. J.-É. Pin, P. Weil: Polynomial closure and unambiguous product. Theory Comput. Systems 30 (1997), 383-422.

11. T. Place: Separating regular languages with two quantifier alternations. To appear in Proc. LICS 2015.

12. T. Place, M. Zeitoun: Going higher in the first-order quantifier alternation hierarchy on words. In Proc. ICALP 2014. Lecture Notes in Comput. Sci. 8573 (2014), 342-353. Full version on arXiv: 1404.6832 [cs.FL].

13. J. Reiterman: The Birkhoff theorem for finite algebras. Algebra Universalis 14 (1982), 1-10.

14. J. Rhodes, B. Steinberg: The q-theory of Finite Semigroups. Springer, 2009.

15. M.-P. Schützenberger, On finite monoids having only trivial subgroups, Inform. and Control 8 (1965), 190-194.

16. H. Straubing: Finite semigroup varieties of the form $V * D$. J. Pure Appl. Algebra 36 (1985), 53-94.

17. H. Straubing: Semigroups and languages of dot-depth 2. In Proc. ICALP 1986. Lecture Notes in Comput. Sci. 226 (1986), 416-423.

18. W. Thomas: Classifying regular events in symbolic logic. J. Comput. System Sci. 25 (1982), 360-376. 